\title{
A generic micromachined silicon platform for high-performance RF passive components
}

\author{
Babak Ziaie $\nmid$ and Khalil Najafi \\ $\dagger$ Department of Electrical and Computer Engineering, University of Minnesota, \\ Minneapolis, MN, USA \\ $\ddagger$ Department of Electrical Engineering and Computer Science, \\ Centre for Integrated Microsystems, University of Michigan, Ann Arbor, MI, USA
}

Received 7 December 1999

\begin{abstract}
This paper describes the development of a micromachined silicon platform fabricated using the dissolved wafer process that supports: (1) high self-resonance frequency and quality factor inductors suspended on a dielectric membrane, (2) low-loss thin-film capacitors, and (3) polysilicon resistors. The process uses deep boron diffusion to create silicon anchors, which support a stress compensated dielectric membrane. A thick resist mold is used to gold electroplate the inductor, top capacitor plate, and bonding pads. This platform can be used to build miniature high-performance transceivers or other RF subsystems using either hybrid-attached surface-mount components or flip-chip bonded RF circuits. Using this technique, a Colpitts transmitter with a five-turn dielectric suspended inductor was designed and fabricated. The transmitter oscillates in the frequency band of 275-375 MHz, consumes $200 \mu \mathrm{A}$ when operated continuously and $100 \mu \mathrm{A}$ when amplitude modulated (on-off keying) at a rate of $1 \mathrm{Mbps}$ (50\% duty cycle).
\end{abstract}

\section{Introduction}

Wireless personal communications and instrumentation microsystems are in high demand [1,2]. Their scope of applications is wide and includes consumer electronics, military, manufacturing, biomedical, security, transportation, and environmental monitoring. High-performance lowpower RF transceivers are an integral part of many of these emerging microsystems. The current technological trend is towards using silicon to implement many of the RF subsystems previously realized with compound semiconductor materials [3,4]. This will eventually lead to lower costs, higher levels of integration, and the ability to include both the sensing and actuating components with the signal processing and RF circuitry. Recent improvements in $\mathrm{Si} / \mathrm{SiGe}$ heterojunction bipolar transistors (HBT) have pushed their cutoff frequencies to beyond $200 \mathrm{GHz}[3,5]$. In addition, the state-of-the-art bulk CMOS technology can offer a viable option for RF designs into the $2 \mathrm{GHz}$ range [4]. However, integration of highperformance lumped or distributed passive components on the silicon substrate remains a challenging task due to the substrate loss and various parasitics [6]. For example, the limiting factors in achieving integrated inductors with high self-resonance frequency and quality factor fabricated on silicon are: substrate and metal series resistance loss (limiting the $Q$ ), and parasitic capacitance to the substrate (reducing the self-resonance frequency and the $Q$ ). Various techniques have been used to overcome these shortcomings, including: (1) selective removal of the substrate under the inductor [7], (2) using thick dielectric layers [8], (3) multilevel metallization [9, 10], and (4) electroplating [11]. Incorporating these techniques into integrated circuits requires added process complexity associated with multilevel metallization, thick dielectric deposition, and front-side etching which is dependent on crystal orientation.

In this paper, we present the development and application of a generic micromachined silicon platform that supports high-performance lumped-element RF passive components. These include: high self-resonance frequency and quality factor inductors, low-loss thin-film capacitors, and polysilicon resistors. With proper design, these components can be used at frequencies up to about $20 \mathrm{GHz}$ where the lumped-element approximation is still valid (i.e. the dimensions $<\lambda / 10$ ). Section 2 describes the overall structure of the silicon platform followed by the component design and models in section 3 . Section 4 discusses the fabrication process followed by the measurement and test results in section 5. Finally, section 6 draws some conclusions from the results of this work.

\section{Overall platform structure}

Figure 1 shows the micromachined silicon platform mounted on a glass back-plate for mechanical support. As can be seen, all the passive elements are fabricated on the silicon substrate and electroplated bonding pads are provided for 


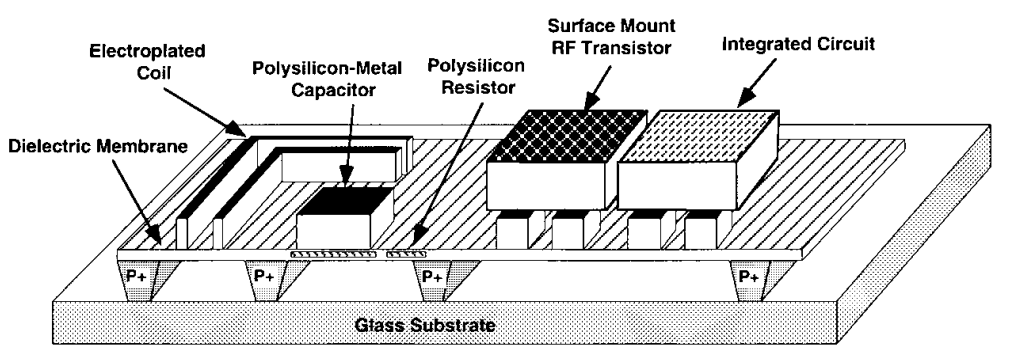

Figure 1. Micromachined silicon platform with surface mount RF transistor, RF integrated circuit, and glass back-plate support.

attachment of discrete active components or flip-chip bonded RF circuits. This platform is fabricated by the dissolved wafer process (see section 4), which uses a deep boron diffusion step to create silicon anchors supporting a stress compensated dielectric membrane. Various passive components including: (1) high self-resonant frequency and quality factor inductors, (2) low-loss thin-film capacitors, and (3) polysilicon resistors, are fabricated on top of the membrane. This technique is simple, saves area, alleviates the need for front-side etching, and does not require multilevel metallization. In addition, various components can be optimized independently for the required application. The capacitive parasitics associated with the surface mount device pads can also be minimized by suspending them on the membrane. The removal of the silicon from underneath the inductor reduces the substrate loss and parasitic capacitances to the substrate. This, in addition to electroplating a thick gold layer, increases the quality factor and self-resonance frequency of the inductor. Thin-film capacitors can achieve low loss through the deposition of high-quality LPCVD dielectric layers and lowresistivity metal plates. Polysilicon resistors can be used to design high-value resistors in low-power applications. This platform can be used to build miniature transceivers or other RF subsystems, either stand-alone to support hybrid attached surface-mount transistors, or flip-chip bonded to RF circuits on an integrated chip, thus saving valuable area.

\section{Components design and models}

In order to accurately design and predict the behavior of various passive components, lumped-element circuit models were used. Figure 2 shows the physical models used to design the inductor, and the capacitor [12]. The inductor is modeled by $L_{s}$, representing the low-frequency inductance, $R_{s}$ modeling the series resistance having a frequency dependence related to the skin effect and other high frequency effects, $C_{o}$ and $C_{o x}$ representing the fringing and the metal layer to the substrate capacitances, and finally $R_{\text {sub }}$ and $C_{\text {sub }}$ modeling the substrate resistance (due to the eddy currents) and capacitance. Removing the semiconducting substrate from underneath the inductor eliminates the effects of $C_{o x}$, $C_{s u b}$, and $R_{s u b}$. Therefore, one only needs to optimize the values of $L_{s}, R_{s}$, and $C_{o}$ in order to achieve the required selfresonance frequency and quality factor. Figure 3 shows the layout geometry and cross section of the coil. Equation (1) can be used to calculate the low-frequency inductance of an air-core rectangular planar inductor [13].

$$
L \approx \frac{45 \mu_{o} n^{2} a^{2}}{22 r-14 a} \text {. }
$$
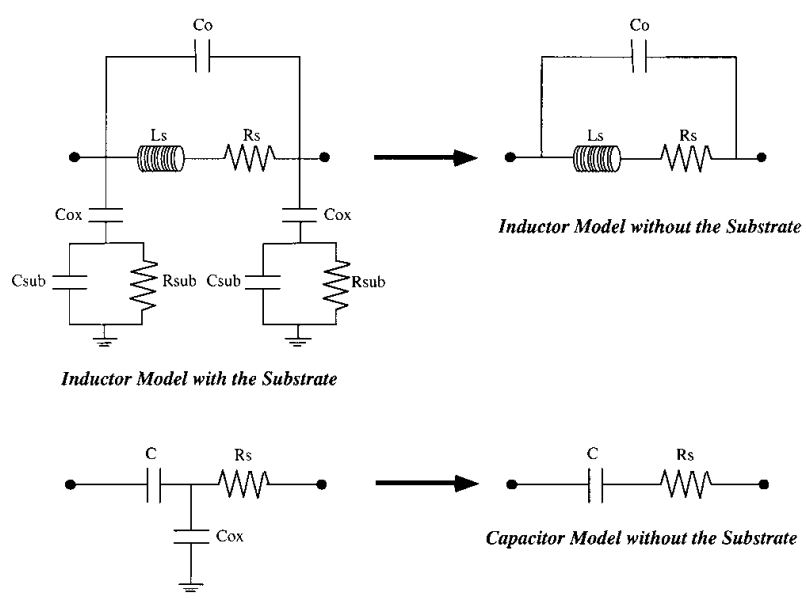

Capacitor Model with the Substrate

Figure 2. Lumped element circuit models for an inductor and a thin-film capacitor with and without the substrate.
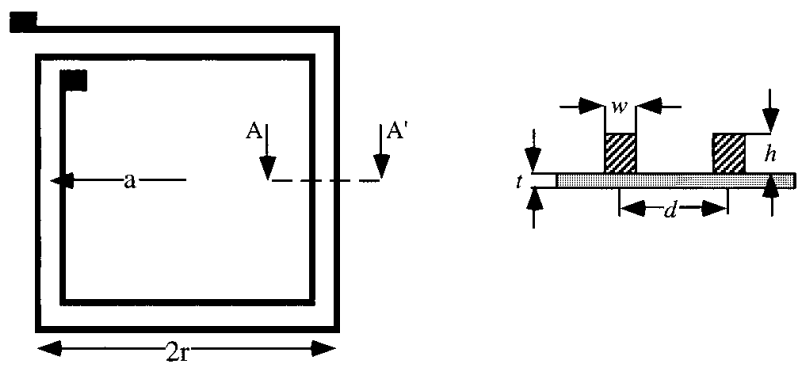

Figure 3. Layout geometry and cross section of an integrated coil.

In this equation, $\mu_{o}$ is the permeability, $n$ is the number of turns, $a$ is the square spiral's mean radius, and $r$ is the outer radius of the spiral (see figure 3). Measurement and simulation results indicate that the formula is accurate to within $5 \%$, which is adequate for most applications [14]. The series resistance $R_{S}$ includes a dc and a frequency dependent component. The dc component can be easily calculated by knowing the coil dimensions and metal resistivity. The frequency dependent component has to account for the skin effect (equation (2)), and magnetic fields. The combined effects can be modeled by equation (3) [15].

$$
\begin{gathered}
\delta=\sqrt{\frac{2 \rho}{\omega \mu}} \\
R_{s}=\frac{\rho l}{w \delta\left(1-\mathrm{e}^{-h / \delta}\right)}
\end{gathered}
$$




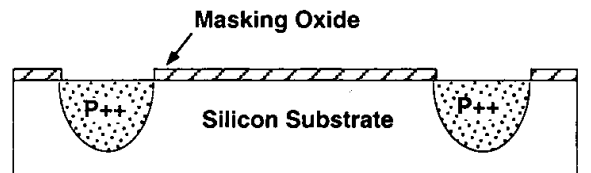

a) Pattern masking oxide and perform a deep boron diffusion.

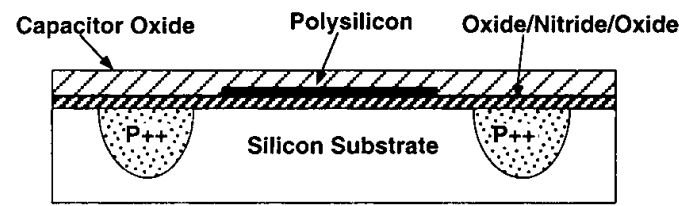

b) Deposit Oxide/Nitride/Oxide, deposit/pattern Polysilicon, deposit and pattern the capacitor oxide.

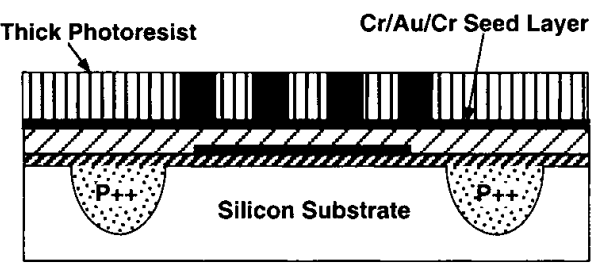

c) Deposit electroplating seed layer ( $\mathrm{Cr} / \mathrm{Au} / \mathrm{Cr}$ ), pattern thick resist, electroplate gold, remove thick resist and strip the plating base.

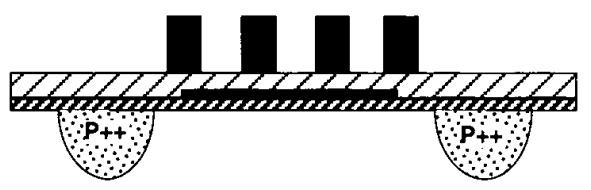

d) Etch silicon in EDP and separate the released substrates.

Figure 4. Cross section of the silicon platform fabrication sequence.

where $\rho$ is the metal resistivity, $\mu$ is the permeability, $\omega$ is the angular frequency, $l$ is the length, $w$ is the line width, $h$ is the metal thickness, and $\delta$ is the metal skin depth. The fringing capacitor between the electroplated turns determines the resonant frequency and can be calculated by using equation (4) (this is the capacitance per unit length) [16]. In this equation, $h$ is the metal thickness, $w$ is the line width, $d$ is the center-to-center distance of adjacent lines, and $\varepsilon_{\text {reff }}$ is the effective dielectric constant $\left(\varepsilon_{\text {reff }} \sim 1\right.$ for $d / t \gg 1$ and $\varepsilon_{\text {reff }}=\left(1+\varepsilon_{r}\right) / 2$ for $d / t \sim 1$, where $t$ is the dielectric thickness).

$$
\frac{C_{o}}{l}=\frac{27.8 \varepsilon_{r e f f}}{\ln \left(\frac{\pi(d-w)}{w+h}+1\right)}\left(\mathrm{pF} \mathrm{m}^{-1}\right) .
$$

The thin-film capacitor is modeled by $C$ representing the low frequency capacitance, $R_{S}$ modeling the effective series resistance, and $C_{o x}$ representing the capacitance from the bottom electrode to the silicon substrate (see figure 2). The effect of $C_{o x}$ is eliminated by removing the substrate. The effective series resistance represents a combination of the dielectric loss, and the resistance in the capacitor electrodes and leads. The driving point impedance $(Z)$ and dissipation factor $(D)$ of the capacitor can be written as:

$$
\begin{gathered}
Z=\frac{\tan \delta}{\omega C}+R_{s}-\frac{j}{\omega C} \\
D=\omega R_{s} C
\end{gathered}
$$

where $\tan \delta$ is the dielectric loss and $R_{s}$ is the equivalent electrode and lead resistance. The dielectric loss is the dominant loss mechanism at low frequencies, whereas at high frequencies the lead and electrode resistances dominate. In many RF applications the equivalent series resistance has to be rather low (e.g. $<1 \Omega$ ). This can be achieved by process modification (i.e. using low sheet resistance metals) and/or layout techniques (multiple capacitors in parallel or minimum resistance patterns) [17]. This is particularly an important consideration if polysilicon is used as the bottom electrode material.

\section{Fabrication process}

Figure 4 shows the fabrication sequence of the silicon platform. The process starts with a deep boron diffusion step $\left(1175^{\circ} \mathrm{C}\right.$ for $7 \mathrm{~h}+1200^{\circ} \mathrm{C}$ drive-in for $5 \mathrm{~h}$ ) to form the support anchors (10 $\mu \mathrm{m}$ deep) for the substrate. This is followed by the deposition of a stress relieved LPCVD dielectric sandwich layer $\mathrm{SiO}_{2}(4000 \AA) / \mathrm{Si}_{3} \mathrm{~N}_{4}(2000 \AA) / \mathrm{SiO}_{2}(4000 \AA)$, which will form the membrane over which the integrated coil and transistor mounting pads are suspended. Next a polysilicon layer is deposited, doped (10-15 $\Omega$ /square), and patterned to define the resistor and the bottom plate of the capacitors. The capacitor dielectric LPCVD oxide $(\sim 5000 \AA)$ is then deposited and contacts are opened to the polysilicon layer. A $\mathrm{Cr}(400 \AA) / \mathrm{Au}(3000 \AA) / \mathrm{Cr}(200 \AA)$ electroplating seed layer is then deposited followed by patterning a thick photoresist (PR4620, spin at $2500 \mathrm{rpm}, 250 \mathrm{~s}$ exposure time, $8 \mathrm{~min}$ development, $\sim 9 \mu \mathrm{m}$ thickness) mold layer. The coil and top capacitor plates are electroplated $\left(55^{\circ} \mathrm{C}\right.$ plating bath, $2 \mathrm{~mA} \mathrm{~cm}^{-2}$ current density) and the seed layer is etched after removing the resist mold. Finally, the wafer is etched in ethylene diamine pyrocatechol (EDP) [18] and individual dies are separated. Depending on the application and design, the dies can be attached with epoxy to a glass back-plate for mechanical support and discrete RF components or integrated RF chips connected to the substrate with silver epoxy or the flip-chip bonding technique (the latter requires an additional solder paste placement step). The micromachined platform is rugged and its fabrication does not depend on the silicon crystal orientation for selective substrate removal. It can support active hybrid components while reducing the large capacitances associated with their bond pads by suspending them on dielectric membranes. Figure 5 shows a photograph of the fabricated substrate with on-chip-electroplated coil, thin-film capacitors, and polysilicon resistors. Figure 6 is an SEM of the cross section of a $6 \mu \mathrm{m}$ electroplated coil, suspended on the dielectric membrane. 


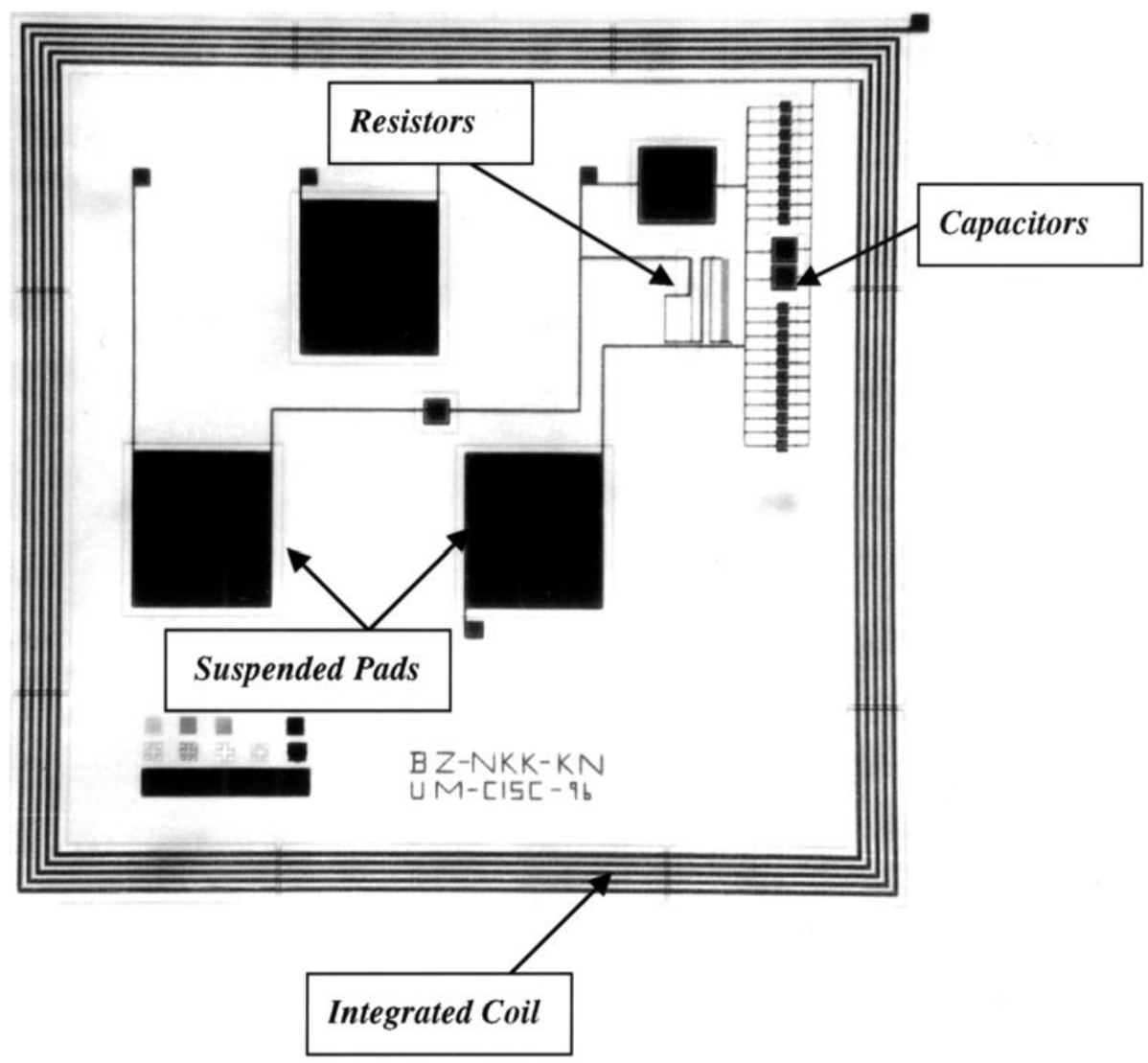

Figure 5. Photograph of a fabricated platform with an on-chip coil, thin-film capacitances and polysilicon resistors.

\section{Test results}

We have used this platform to design and implement a Colpitts oscillator. The oscillator is the transmitter part of a biotelemetry microsystem designed for long-term recording of a number of physiological parameters, figure 7 . The recorded and digitized physiological data is used to amplitude modulate (on-off switching) the Colpitts transmitter. The transmitter is built by attaching a surface mount hybrid RF transistor on the silicon platform. An inductor with a highquality factor reduces the power consumption and phasenoise in the Colpitts transmitter [19]. Equation (7) describes the oscillation condition (loop gain $>1$ ):

$$
g_{m} Q^{2} r_{s} \frac{C_{1}}{C_{1}+C_{2}} \geqslant 1
$$

where $g_{m}\left(I_{c} / V_{T}\right)$ is the transconductance, $Q$ is the quality factor of the coil, $r_{s}$ is the series resistance of the coil, and $C_{1} / C_{1}+C_{2}$ is the capacitance feedback ratio. As can be seen, a high $Q$ allows a reduction in the quiescent current of the transistor. In order to design the transmitter, one starts with the desired current consumption and designs the coil (dimension, number of turns, conductor width spacing and thickness) in order to achieve the required $Q$ and satisfy the oscillation condition. Capacitors $C_{1}$ and $C_{2}$ are next chosen to set the transmission frequency. Many iterations might be necessary to optimize the design. Capacitor $C_{1}$ is trimmable to allow fine-tuning of the oscillation frequency. Emitter resistance $(R)$ along with the driving voltage set the power consumption. Figure 8 shows a SEM of a transmitter chip with electroplated coil and surface mount RF transistor.

Following the platform fabrication, important parameters for various passive components were measured using an RF impedance analyzer (HP4195A). Equivalent series resistance for a typical thin-film polysilicon-metal capacitor was measured to be $\sim 3 \Omega$ at $300 \mathrm{MHz}$. Multiple capacitors connected in parallel were used in the actual transmitter circuitry to reduce the series resistance to below $1 \Omega$. A fiveturn inductor with $25 \mu \mathrm{m}$ linewidth and separation, $5 \mu \mathrm{m}$ metal thickness, and $2.5 \mathrm{~mm}$ outer radius was designed for the transmitter coil. The inductance and the quality factor for the coil were measured at $300 \mathrm{MHz}$ before and after the substrate removal. The measured inductance was $350 \mathrm{nH}$ in both cases, which was within $5 \%$ of the designed value $(370 \mathrm{nH}$ using equation (1)). However, the quality factor showed a dramatic increase from 3 to 18 after the substrate removal. This clearly demonstrates the effectiveness of removing the substrate in reducing the eddy current losses and increasing the quality factor. Table 1 summarizes the measurement results for an integrated coil and capacitor suspended on a dielectric membrane.

Following the characterization of passive components, a surface mount RF transistor (Motorola MMBR931LT1) was mounted on the substrate using silver epoxy and the transmitter was operated with a $3 \mathrm{~V}$ battery. Figure 9 shows the output spectrum of a transmitter being switched at $1 \mathrm{Mbps}$. As can be seen, the oscillation frequency is at $\sim 315 \mathrm{MHz}$ and 

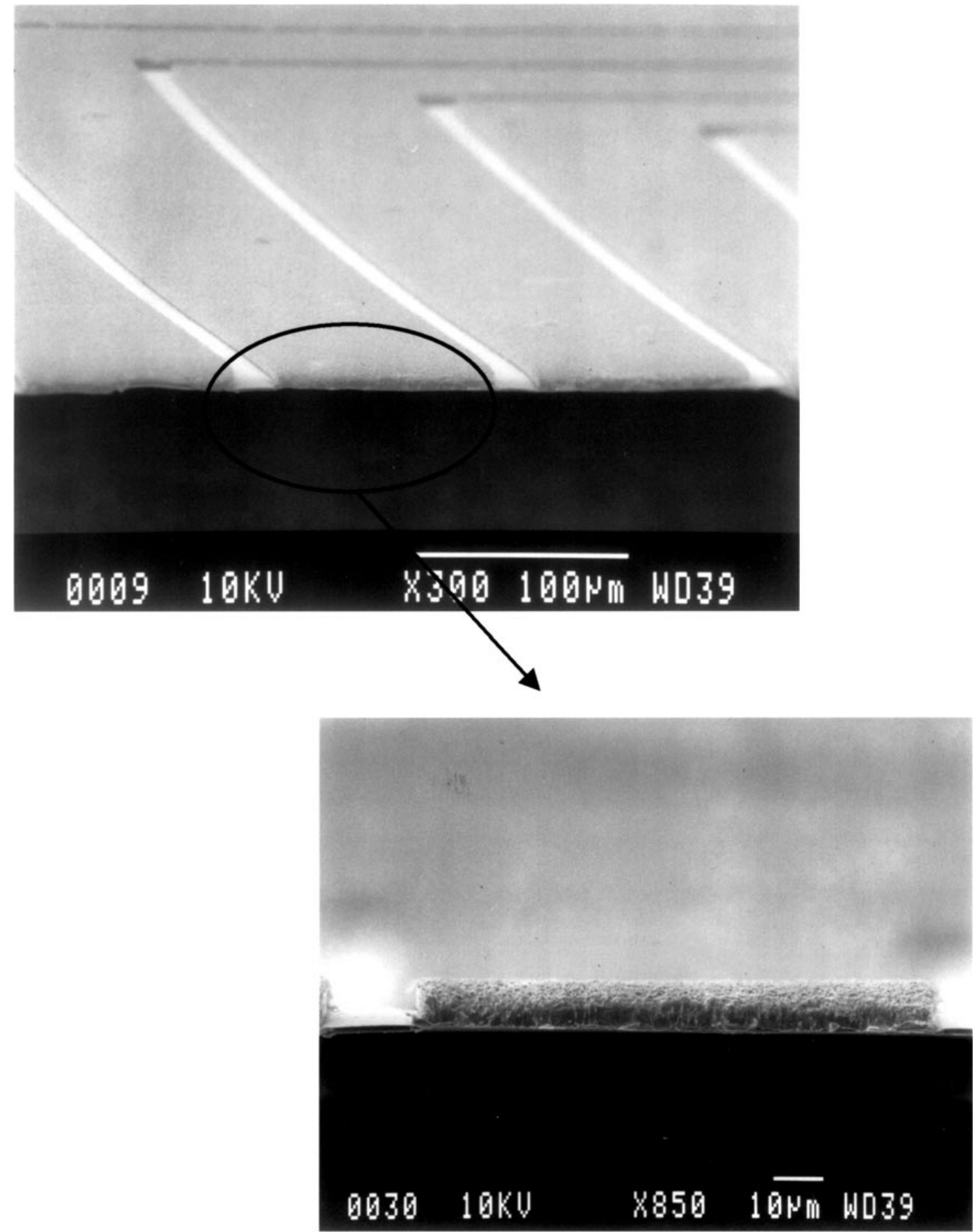

Figure 6. SEM of the cross section of a $6 \mu \mathrm{m}$ electroplated coil suspended on the dielectric membrane.

Table 1. The measurement results for an integrated coil and capacitor suspended on a dielectric membrane.

\begin{tabular}{ll}
\hline Component & Measured value at $300 \mathrm{MHz}$ \\
\hline Coil & $350 \mathrm{nH}$ \\
$L_{o}$ & $36 \Omega$ \\
$R_{S}(\mathrm{AC})$ & 18 \\
$Q($ at $300 \mathrm{MHz})$ & $>500 \mathrm{MHz}$ \\
$f_{r s}$ & \\
Capacitors & $6.9 \times 10^{-9} \mathrm{~F} \mathrm{~cm}^{-2}$ \\
$C /$ area & $3 \Omega$ \\
Equivalent series resistance & 0.015 \\
Dissipation factor & \\
\hline
\end{tabular}

the spectrum is spread according to the incoming pulse rate and duty cycle. The transmitter consumes $\sim 100 \mu \mathrm{A}$ and has a transmission range of $\sim 3 \mathrm{ft}$. The power consumption in the pulsed mode of operation is determined by the emitter resistance and the driver pulse amplitude and duty cycle (one can reduce the current drain either by lowering the pulse amplitude, $\sim 60 \mu \mathrm{A}$ if the pulse amplitude is $2 \mathrm{~V}$, or using a lower duty cycle pulse stream). Table 2 summarizes important characteristics and test results of the Colpitts transmitter. 


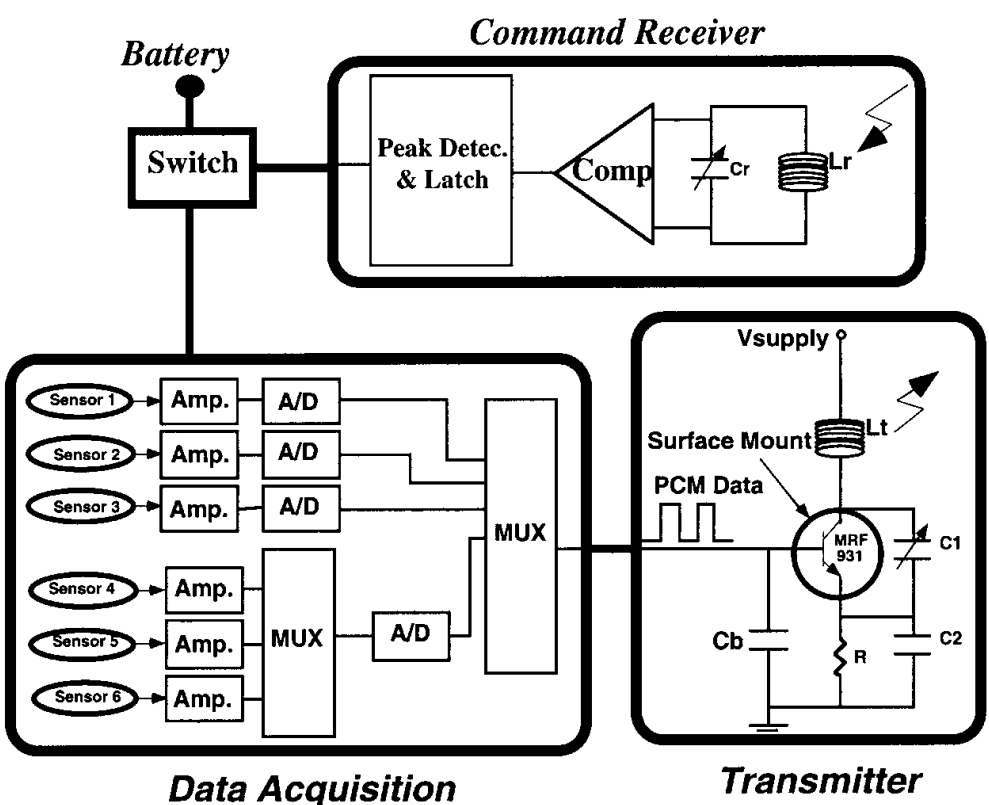

Figure 7. Biotelemetry microsystem with Colpitts transmitter for measuring multiple physiological parameters.

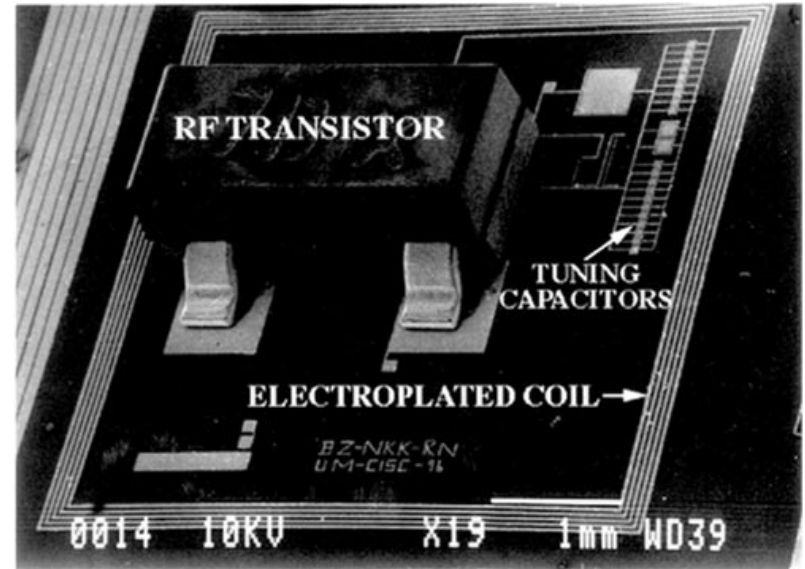

Figure 8. SEM of a transmitter platform with a surface mount RF transistor.

Table 2. Important characteristics and test results of the Colpitts transmitter.

\begin{tabular}{ll}
\hline Area & $5 \times 5 \mathrm{~mm}^{2}$ \\
Power consumption & $200 \mu \mathrm{A}$ continuous/100 $\mu \mathrm{A}$ \\
& at $1 \mathrm{Mbps}$ data rate \\
Range & $\sim 3 \mathrm{ft}$ \\
Operating frequency & $275-375 \mathrm{MHz}$ \\
Modulation rate & up to $1 \mathrm{Mbps}$ \\
\hline
\end{tabular}

\section{Conclusion}

We have developed a generic micromachined silicon platform for high-performance RF passive components. This platform is fabricated by the dissolved wafer process and supports: (1) high self-resonant frequency and quality factor inductors suspended on a dielectric membrane, (2) low-loss thin-film capacitors, and (3) polysilicon resistors. By suspending an electroplated coil on a dielectric membrane, we were able to increase the self-resonance frequency and the quality

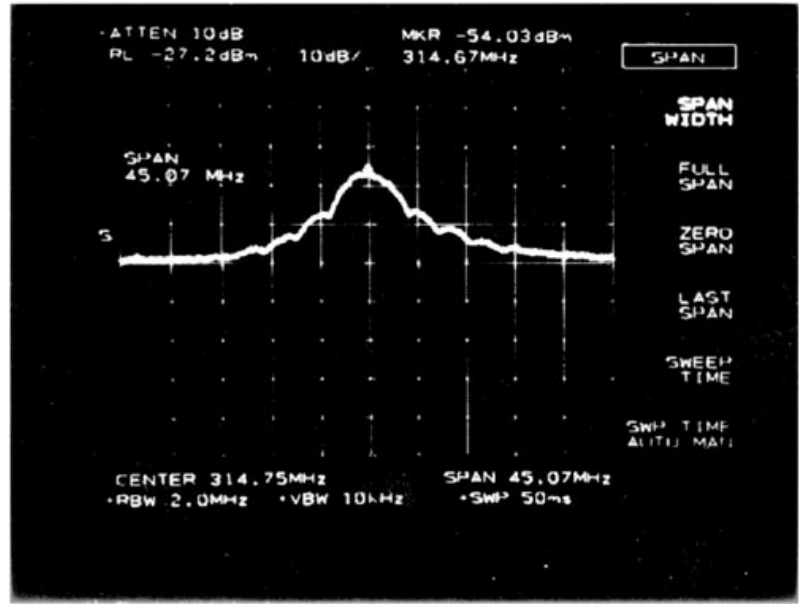

Figure 9. The output spectrum of a transmitter operating at $\sim 315 \mathrm{MHz}$ and being modulated at $1 \mathrm{Mbps}$.

factor of the inductor without using multilevel metallization and front-side selective substrate removal which adds to the process complexity. This technique allows full optimization of various passive components without the need for any modifications to the foundry available CMOS or BiCMOS circuitries. This platform can be used to build miniature, lightweight, high-performance (low-power and low-noise) transceivers or other RF subsystems using hybrid-attached surface-mount or flip-chip bonded RF circuits.

\section{Acknowledgments}

The authors would like to thank Mr John W Hines and Dr Chris J Somps of the NASA Ames Research Center for their support and encouragement. We would also like to thank Professor David J Anderson of the University of Michigan and Mr Namik Kocaman of Level One Communications 
Inc. for their technical contributions to this article. This work was supported by the National Aeronautics and Space Administration (NASA), under grant NAWG-4494.

\section{References}

[1] Gray P R and Meyer R G 1995 Future directions in silicon IC's for RF personal communications Proc. Custom Integrated Circuits Conf. pp 83-9

[2] Mason A, Yazdi N, Chavan A V, Najafi K and Wise K D 1998 A generic multielement microsystem for portable wireless applications Proc. IEEE 86 1733-46

[3] Luy J F et al $1995 \mathrm{Si} / \mathrm{SiGe}$ MMIC's IEEE Trans. Microwave Theory Techniques $\mathbf{4 3}$ 705-14

[4] Voinigescu S P, Tarasewiscz S W, MacElwee T and Ilowski J 1995 An assessment of the state-of-the-art $0.5 \mu \mathrm{m}$ bulk CMOS technology for RF applications Proc. IEDM pp 721-4

[5] Harame D L et al 1994 A 200 mm SiGe-HBT technology for wireless and mixed-signal applications Proc. IEDM pp 437-40

[6] Warner R M 1965 Integrated Circuits: Design Principles and Fabrication (New York: McGraw-Hill)

[7] Chang J Y C, Abidi A A and Gaitan M 1993 Large suspended inductors on silicon and their use in a $2-\mu \mathrm{m}$ CMOS RF amplifier IEEE Electron Device Lett. 14 246-8

[8] Case M 1997 SiGe MMICs and flip-chip MICs for low cost microwave systems Microwave J. 40 264-76
[9] Burghartz J N et al 1995 High-Q inductors in standard silicon interconnect technology and its application to an integrated RF power amplifier Proc. IEDM pp 1015-17

[10] Ashby K B et al 1996 High Q inductors for wireless applications in a complementary silicon bipolar process IEEE J. Solid-State Circuits 31 4-9

[11] Watanabe Y et al 1995 A new fabrication process of a planar coil using photosensitive polyimide and electroplating Proc. Transducers '95 pp 268-71

[12] Long J R and Copeland M A 1996 The modeling, characterization, and design of monolithic inductors for silicon RF IC's IEEE J. Solid-State Circuits 32 357-69

[13] Wheeler H A 1928 Simple inductance formulas for radio coils IRE Proc. p 1398

[14] Lee T H 1998 The Design of CMOS Radio-Frequency Integrated Circuits (Cambridge: Cambridge University Press)

[15] Yue C P, Ryu C, Lee T H and Wong S S 1996 A physical model for planar spiral inductors on silicon Proc. IEDM pp 155-8

[16] Walker C S 1990 Capacitance, Inductance and Crosstalk Analysis (Boston: Artech House)

[17] Glaser A B and Subak-Sharpe G E 1977 Integrated Circuit Engineering (Reading, MA: Addison-Wesley)

[18] Raley N F, Ugiyama Y and Van Duzer T 1984 (100) Silicon etch rate dependence on boron concentration in ehtylenediamine-pyrocatechol-water solution J. Electrochem. Soc. 131161

[19] Rhea R W 1997 Oscillator Design and Computer Simulation (New York: McGraw-Hill) 